\title{
A note on value distribution of nonhomogeneous differential polynomials
}

(Dedicated to Prof. B.K. Lahiri on his 70th birth anniversary)

\section{Indrajit LAHIRI}

(Received March 26, 2001; Revised June 6, 2001)

\begin{abstract}
In the note we prove a result on value distribution of nonhomogeneous differential polynomials which improves a long standing theorem of C.C. Yang.
\end{abstract}

Key words: differential polynomial, value distribution.

\section{Introduction and Definitions}

Let $f$ be a transcendental meromorphic function in the open complex plane $\mathbb{C}$. The problem of investigating possible Picard values of the derivative of $f$ leads to the problem of investigating the value distribution of certain polynomials in $f$ and its derivatives which are called differential polynomials generated by $f$ and is explained in Definition 2 .

Definition 1 A meromorphic function $a$ is said to be a small function of $f$ if $T(r, a)=S(r, f)$.

Definition $2[1,3]$ Let $n_{0 j}, n_{1 j}, \ldots, n_{k j}$ be nonnegative integers. The expression $M_{j}[f]=b_{j}(f)^{n_{0 j}}\left(f^{(1)}\right)^{n_{1 j}} \cdots\left(f^{(k)}\right)^{n_{k j}}$ is called a differential monomial generated by $f$ of degree $\gamma_{M_{j}}=\sum_{i=0}^{k} n_{i j}$ and weight $\Gamma_{M_{j}}=\sum_{i=0}^{k}(i+$ 1) $n_{i j}$, where $T\left(r, b_{j}\right)=S(r, f)$.

The sum of the monomials $P[f]=\sum_{i=1}^{l} M_{j}[f]$ is called a differential polynomial generated by $f$ of degree $\gamma_{P}=\max \left\{\gamma_{M_{j}}: 1 \leq j \leq l\right\}$ and weight $\Gamma_{P}=\max \left\{\Gamma_{M_{j}}: 1 \leq j \leq l\right\}$.

The numbers $\underline{\gamma}_{P}=\min \left\{\gamma_{M_{j}}: 1 \leq j \leq l\right\}$ and $k$ (the highest order of the derivative of $f$ in $P[f]$ ) are called respectively the lower degree and order of $P[f]$.

$P[f]$ is said to be homogeneous if $\gamma_{P}=\underline{\gamma}_{P}$.

Also we denote by $\gamma_{P}^{*}$ the number $\gamma_{P}^{*}=\max \left\{\gamma_{M_{j}}: \gamma_{M_{j}}<\gamma_{P}\right.$ and $1 \leq j \leq l\}$. 
Definition 3 For a complex number $a \in \mathbb{C} \cup\{\infty\}$ we denote by $N_{1)}(r, a ; f)$ the counting function of simple $a$-points of $f$.

We do not explain the standard definitions and notations of the value distribution theory because those are available in [6]. Hayman [5] proved the following theorems.

Theorem $\mathbf{A}$ If $f$ is transcendental entire and $n \geq 3, a \neq 0$ then $\psi=$ $f^{\prime}-a(f)^{n}$ assume all finite values infinitely often.

Theorem B If $f$ is transcendental entire and $n \geq 2$ then $f^{\prime}(f)^{n}$ assumes all finite values except possibly zero infinitely often.

Clunie [2] proved Theorem B for $n \geq 1$. Later on Sons [7] generalised Theorem $\mathrm{B}$ and proved the following result.

Theorem C If $f$ is transcendental entire and $\psi=(f)^{n_{0}}\left(f^{(1)}\right)^{n_{1}} \ldots$ $\left(f^{(k)}\right)^{n_{k}}$, where $n_{0} \geq 2, n_{k} \geq 1$ and $n_{i} \geq 0$ for $i \neq 0, k$ then $\delta(a ; \psi)<1$ for $a \neq 0, \infty$. Moreover if $N_{1)}(r, 0 ; f)=o\{T(r, f)\}$ as $r \rightarrow \infty$ then for $n_{0} \geq 1$ the same conclusion holds.

For differential polynomials C.C. Yang [8] proved the following theorem. Theorem D Let $f$ be transcendental meromorphic with $N(r, f)+$ $N(r, 0 ; f)=S(r, f)$ and $\psi=\sum a(f)^{p_{o}}\left(f^{(1)}\right)^{p_{1}} \cdots\left(f^{(k)}\right)^{p_{k}}$ with no constant term where $T(r, a)=S(r, f)$. If the degree $n$ of $\psi$ is greater than one and $p_{0}<n, 0 \leq p_{i} \leq n$ for all $i \neq 0$, then $\delta(b, \psi)<1$ for all $b \neq 0, \infty$.

Following theorem of Gopalakrishna and Bhoosnurmath [4] shows that for a homogeneous differential polynomial we get a better result.

Theorem E Let $f$ be meromorphic with $\bar{N}(r, f)+\bar{N}(r, 0 ; f)=S(r, f)$ and $\psi(f)$ be a nonconstant homogeneous differential polynomial. Then $\Theta(b ; \psi)=$ 0 for all $b \neq 0, \infty$.

However for nonhomogeneous differential polynomials Theorem $\mathrm{E}$ does not hold. For, let $f=\exp (z), \psi=f^{2}-2 i f^{\prime}$. Then $\Theta(1 ; \psi)=\frac{1}{2}$.

For nonhomogeneous differential polynomials C.C. Yang [9] proved the following theorem.

Theorem $\mathbf{F}$ Let $f$ be a transcendental meromorphic function with $N(r, f)+N(r, 0 ; f)=S(r, f)$. Let $\psi(f)$ be a differential polynomial in $f$ of degree $n \geq 2$ such that all the terms of $\psi(f)$ have degree at least two. 
If $\psi(f)$ is nonhomogeneous then $\delta(b, \psi) \leq 1-\frac{1}{2 n}$ for all $b \neq \infty$.

Now one may naturally ask: Is the upper bound $1-\frac{1}{2 n}$ in Theorem $\mathrm{F}$ sharp? If not, what is the best possible upper bound?

The purpose of the note is to study this problem. We apply a result of H.X. Yi [10] to prove a theorem on the value distribution of nonhomogeneous diferential polynomials which not only gives the best possible upper bound for $\delta(b ; P[f])$ in Theorem $\mathrm{F}$ but also estimate a larger quantity, the ramification index, under weaker hypothesis.

\section{Lemmas}

In this section we state two lemmas which will be needed in the sequel.

Lemma $1[9] \quad$ Let $P[f]=\sum_{i=0}^{n} a_{i} f^{i}$ where $a_{n} \not \equiv 0$ and $T\left(r, a_{i}\right)=S(r, f)$ for $i=0,1,2, \ldots, n$. Then $T(r, P[f])=n T(r, f)+S(r, f)$.

Lemma $2[10]$ Let $F=f^{n}+P[f]$, where $n \geq 2$ and $\Gamma_{P} \leq n-1$. Then either $P[f] \equiv 0$ or

$$
\begin{aligned}
\left(n-\gamma_{P}\right) T(r, f) \leq & \bar{N}(r, 0 ; f)+\bar{N}(r, 0 ; F) \\
& +\left(1+\Gamma_{P}-\gamma_{P}\right) \bar{N}(r, \infty ; f)+S(r, f) .
\end{aligned}
$$

\section{The Main Result}

In this section we prove the main result of the note.

Theorem 1 Let either $\Gamma_{M_{j}}=\gamma_{M_{j}}(j=1,2, \ldots, l)$ or $\bar{N}(r, 0 ; f)+$ $\bar{N}(r, f)=S(r, f)$ and $P[f]=\sum_{j=1}^{l} M_{j}[f]$ be such that $\gamma_{P}>\underline{\gamma}_{P} \geq 1$. Then

$$
\Theta(a ; P[f]) \leq \frac{\gamma_{P}^{*}}{\gamma_{P}}
$$

for any small function $a(\not \equiv \infty)$ of $f$.

Proof. Clearly we can write $M_{j}[f]=c_{j} f^{\gamma_{M_{j}}}$, where

$$
c_{j}=b_{j}\left(\frac{f^{(1)}}{f}\right)^{n_{1 j}}\left(\frac{f^{(2)}}{f}\right)^{n_{2 j}} \cdots\left(\frac{f^{(k)}}{f}\right)^{n_{k j}} .
$$

Now by Milloux theorem $\{$ p.55[6] $\}$ we see that 


$$
m\left(r, c_{j}\right) \leq m\left(r, b_{j}\right)+\sum_{i=1}^{k} n_{i j} m\left(r, \frac{f^{(i)}}{f}\right)=S(r, f)
$$

Also

$$
N\left(r, c_{j}\right) \leq N\left(r, b_{j}\right)+\sum_{i=1}^{k} n_{i j} N\left(r, \frac{f^{(i)}}{f}\right) .
$$

We note that poles of $\frac{f^{(i)}}{f}$ occur only at the poles and zeros of $f$ and a pole or a zero of $f$ is a pole of $\frac{f^{(i)}}{f}$ with multiplicity at most $i$. So

$$
N\left(r, \frac{f^{(i)}}{f}\right) \leq i\{\bar{N}(r, f)+\bar{N}(r, 0 ; f)\} .
$$

Therefore

$$
\begin{aligned}
N\left(r, c_{j}\right) & \leq\left\{\sum_{i=1}^{k} i n_{i j}\right\}\{\bar{N}(r, f)+\bar{N}(r, 0 ; f)\}+S(r, f) \\
& =\left(\Gamma_{M_{j}}-\gamma_{M_{j}}\right)\{\bar{N}(r, f)+\bar{N}(r, 0 ; f)\}+S(r, f) \\
& =S(r, f),
\end{aligned}
$$

by the given condition. Hence $T\left(r, c_{j}\right)=S(r, f)$ for $j=1,2, \ldots, l$.

Now collecting the same powers of $f$ together and if necessary putting some $\alpha_{i} \equiv 0$

$$
P[f]=\alpha_{\gamma_{P}} f^{\gamma_{P}}+\sum_{i=1}^{\gamma_{P}^{*}} \alpha_{i} f^{i}
$$

where $T\left(r, \alpha_{i}\right)=S(r, f)$ for $i=1,2, \ldots, \gamma_{P}^{*}, \gamma_{P}$ and $\alpha_{\gamma_{P}} \not \equiv 0$.

Now we put

$$
\begin{aligned}
F & =\frac{P[f]}{\alpha_{\gamma_{P}}}-\frac{a}{\alpha_{\gamma_{P}}} \\
& =f^{\gamma_{P}}+\left\{\sum_{i=1}^{\gamma_{P}^{*}} \frac{\alpha_{i}}{\alpha_{\gamma_{P}}} f^{i}-\frac{a}{\alpha_{\gamma_{P}}}\right\}
\end{aligned}
$$

where $T(r, a)=S(r, f)$ and $a \not \equiv \infty$. 
Clearly

$$
\sum_{i=1}^{\gamma_{P}^{*}} \frac{\alpha_{i}}{\alpha_{\gamma_{P}}} f^{i}-\frac{a}{\alpha_{\gamma_{P}}} \not \equiv 0 .
$$

For, otherwise by Lemma 1 we arrive at a contradiction. Hence by Lemma 2 we obtain

$$
\begin{aligned}
\left(\gamma_{P}-\gamma_{P}^{*}\right) T(r, f) & \leq \bar{N}(r, 0 ; F)+S(r, f) \\
& =\bar{N}(r, a ; P[f])+S(r, f) .
\end{aligned}
$$

Also by Lemma 1 we get from (1) that

$$
T(r, P[f])=\gamma_{P} T(r, f)+S(r, f) .
$$

Therefore it follows from (2) that

$$
\left(1-\frac{\gamma_{P}^{*}}{\gamma_{P}}\right) T(r, P[f]) \leq \bar{N}(r, a ; P[f])+S(r, P[f]),
$$

from which the theorem follows. This proves the theorem.

Remark 1 The condition $\underline{\gamma}_{P} \geq 1$ is necessary. For, let $f=\exp (z)$ and $P[f]=\left(f^{\prime \prime}\right)^{2}+2 f^{\prime}-2 f+1\{$ cf. $[9]\}$. Then $\Theta(1 ; P[f])=1$.

Remark 2 The bound $\gamma_{P}^{*} / \gamma_{P}$ is sharp. For, let $f=\exp (z)$ and $P[f]=$ $f^{3}-f^{2}$. Then $\Theta(0 ; P[f])=2 / 3$.

\section{References}

[1] Bhattacharjee N.R. and Lahiri I., Growth and value distribution of differential polynomials. Bull. Math. Soc. Sc. Math. Roumanie, Tome 39 (87), No.1-4 (1996), 85-104.

[2] Clunie J., On a result of Hayman. J. London Math. Soc. 42 (1967), 389-392.

[3] Doeringer W., Exceptional values of differential polynomials. Pacific J. Math. 98 (1) (1980), 55-62.

[4] Gopalakrishna H.S. and Bhoosnurmath S.S., On the distribution of value of differential polynomials. Indian J. Pure Appl. Math. 17 (3) (1986), 367-372.

[5] Hayman W.K., Picard values of meromorphic functions and their derivatives. Ann. of Math. 70 (1959), 9-42.

[6] Hayman W.K., Meromorphic Functions. The Clarendon Press, Oxford (1964).

[7] Sons L.R., Deficiencies of monomials. Math. Z. 111 (1969), 53-68. 
[ 8] Yang C.C., On deficiencies of differential polynomials. Math. Z. 116 (1970), 197204.

[9] Yang C.C., On deficiencies of differential polynomials II. Math. Z. 125 (1972), 107-112.

[10] Yi H.X., On a theorem of Tumura and Clunie for a differential polynomial. Bull. London Math. Soc. 20 (1988), 593-596.

Department of Mathematics

University of Kalyani

West Bengal 741235

India

E-mail: indrajit@cal2.vsnl.net.in 